\title{
The complex EAS installation of the Tien Shan mountain cosmic ray station
}

\author{
Iskakov Bakhtiyar ${ }^{1}$ \\ Al-Farabi-Kazakh National University, Almaty, Kazakhstan \\ E-mail: leodel@mail.ru
}

\section{Tautayev Yernar}

Al-Farabi-Kazakh National University, Almaty, Kazakhstan

E-mail: tautaevemail.ru

\section{Sadykov Turlan}

Satbayev University, Institute of Physics and Technology, Almaty, Kazakhstan

E-mail: 8896970leodelegmail.com

\section{Khabargeldina Maral}

Al-Farabi-Kazakh National University, Almaty, Kazakhstan

E-mail: 8896970leodelegmail.com

\section{Tastanova Kalamkyz}

Al-Farabi-Kazakh National University, Almaty, Kazakhstan

E-mail: 8896970leodelegmail.com

\begin{abstract}
Tien Shan High-Altitude Scientific Station is located at an altitude of $3340 \mathrm{~m}$ above sea level at a distance of $30 \mathrm{~km}$ from the city of Almaty. The station conducted a wide range of research projects. One of them is the "Hadron-55" complex with the help of which various effects and phenomena associated with the EAS passing through the Earth's atmosphere are studied. The installation includes a network of neutron monitor, ionization calorimeters, a system of scintillation detectors. In addition, the station contains such complexes as ALMAREC, which studies radio signals from EAS and an acoustic recording system that allows recording possible acoustic effects when muons pass through the earth's crust. Scientific station "Space Station" is one of the best in the CIS.
\end{abstract}

36th International Cosmic Ray Conference -ICRC2019-

July 24th - August 1st, 2019

Madison, WI, U.S.A.

${ }^{1}$ Speaker 


\section{Introduction}

The researchers of Physico-Technical Institute study cosmic rays (CR) since the end of the 1950's. The institute has highly qualified researchers, unique Tien Shan and Intermediate scientific stations located at $3340 \mathrm{~m}$ and $1700 \mathrm{~m}$ above the sea level. Modern level of the conducted investigations and high quality of the results derived by the Tien Shan high-mountain scientific station were stipulated by many years of wide and comprehensive cooperation with the Russian Research Institutes and by active involvement in the International scientific projects. Collaboration that exists between Physical Institute after Lebedev of the Russian Academy of Sciences and Tien Shan high-mountain scientific station is realized by virtue of the program of joint investigations between research institutes and universities of Kazakhstan and Russia, as well as the Consortium Agreement on establishing "Eurasian high-mountain scientific Centre of the cosmic rays". Later, Kazakh National University after al-Farabi and Ionosphere Institute joined this agreement.

For tackling more fundamental and applied problems using the station located at $3340 \mathrm{~m}$ above sea level, research teams of the above-mentioned institutes implement research investigations on the following topics:

- searching of structures in the particles distributions from EAS forward cone at high energies on the "Hadron - 55" installation;

- registration and investigation of the radio emission from EAS;

- investigation of the Earth's crust tension degree of the Almaty seismically active region by registering high energy cosmic rays muons.

\section{Results and Discussion}

The structures searching in particles distributions from the forward cone of the extended air showers (EAS) at high energies on the "Hadron - 55" installation. In the context of this problem, the following two unique phenomena that were discovered should be noted:

- events with anomalous relation of charged and neutral components, so-called centaur of the event;

- phenomenon of coplanar particles emission, the events with the geometrical alignment.

Since the problem of primary cosmic rays composition at the super high energies is far from being solved, the initial set of goals that hold for EAS complete installations are as relevant as before. Today, however, this problem can be solved in a more effective way by applying more advanced understanding of this problem and using progress of the last two decades in the field of experimental and computer technologies, thereby permitting to use new methods of experimental data processing and analysis. This very approach was used as a foundation in the "Hadron-55" project, which according to us is capable to make progress in terms of solving classical problems (the primary cosmic rays parameters investigation and building interaction model) that are relevant within the context of EAS complex installations. 


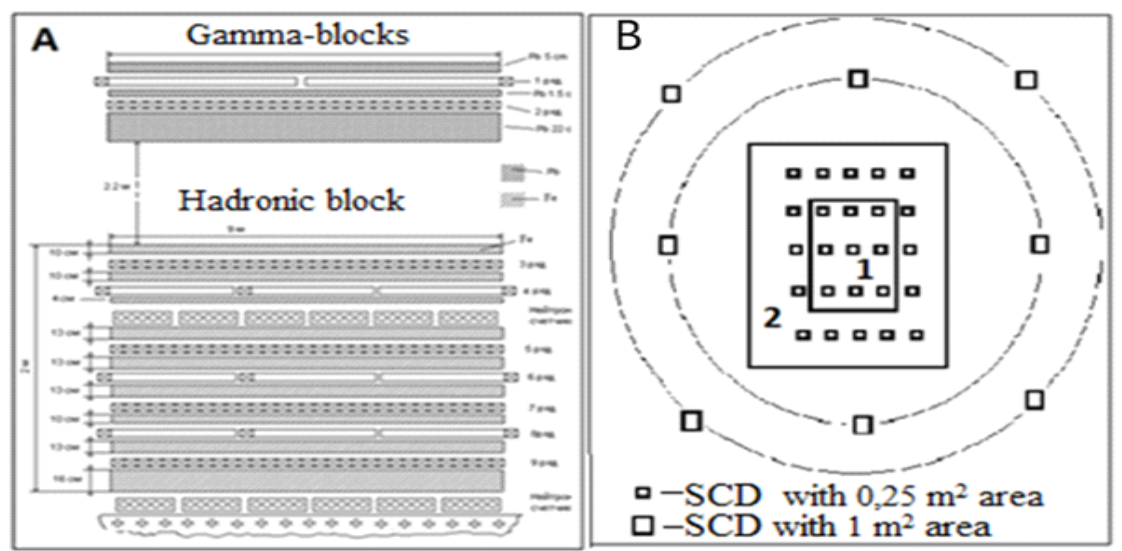

Fig.1. A - ionization calorimeter, composed of gamma-blocks and lower on 2,2 m hadronic block. B -installation detectors disposition circuit (top view): 1-calorimeter disposition in the laboratory housing. 2- SCD by $0.25 \mathrm{~m}^{2}$ area disposition in the laboratory housing. In the closest circle within a radius of $40 \mathrm{~m}$ and in large circle within a radius of $100 \mathrm{~m}$ in fours SCD with $1 \mathrm{~m}^{2}$ area are disposed.

Since the problem of primary cosmic rays composition at the super high energies is far from being solved, the initial set of goals that hold for EAS complete installations are as relevant as before. Today, however, this problem can be solved in a more effective way by applying more advanced understanding of this problem and using progress of the last two decades in the field of experimental and computer technologies, thereby permitting to use new methods of experimental data processing and analysis. This very approach was used as a foundation in the "Hadron-55" project, which according to us is capable to make progress in terms of solving classical problems (the primary cosmic rays parameters investigation and building interaction model) that are relevant within the context of EAS complex installations.

Registration and investigation of the radio emission from EAS. In 2018, work was carried out to modernize the recording of radio emission from extensive air showers on the Almarec installation (Fig. 2). Each antenna station is located at a distance of $30 \mathrm{~m}$ from the registration point in the north, south, west, east direction and has two perpendicular antennas, which allows restored the polarization of the electromagnetic wave.

As a result of the preliminary experiment, some event candidates were selected that demonstrate the presence of a noticeable radio signal pulse in the vicinity of the nearest EAS arrival time 1-3 $\mu \mathrm{s}$. The features of the particle density distribution in these events suggest that most of them have a fairly high primary energy $\mathrm{E}_{0}>(2-5) \cdot 10^{16} \mathrm{eV}$ and the proximity of the shower center to the radio antenna, therefore the coincidence time of the observed radio pulse with the arrival time of the shower front in these events can not be completely random. Consequently, the radio antenna system installed on the Tien Shan, together with the developed software for recording the signal, really provides an effective choice of radio emission from EAS particles, and further work in this direction should be seriously considered. 


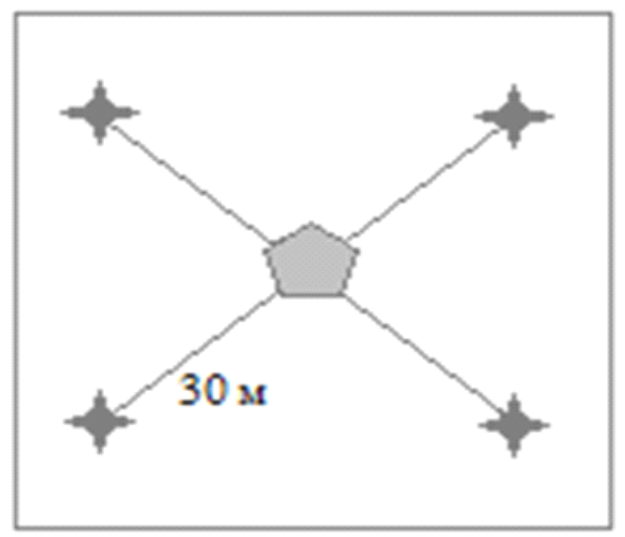

Fig.2. A schematic arrangement of the antennas at a distance of $30 \mathrm{~m}$ from the point of registration.

As a result of the preliminary experiment, some event candidates were selected that demonstrate the presence of a noticeable radio signal pulse in the vicinity of the nearest EAS arrival time 1-3 $\mu \mathrm{s}$. The features of the particle density distribution in these events suggest that most of them have a fairly high primary energy $\mathrm{E}_{0}>(2-5) \cdot 10^{16} \mathrm{eV}$ and the proximity of the shower center to the radio antenna, therefore the coincidence time of the observed radio pulse with the arrival time of the shower front in these events can not be completely random. Consequently, the radio antenna system installed on the Tien Shan, together with the developed software for recording the signal, really provides an effective choice of radio emission from EAS particles, and further work in this direction should be seriously considered.

Investigation of Earth's crust tension degree of Almaty active region by registering cosmic rays muons of high energy. Radiation acoustics is a scientific multidisciplinary area, which is developing between acoustics, nuclear physics and high energy physics. Its foundation is formed by studies and application of radiation-acoustic effects that are nascent at the penetrating radiation interaction with a matter. At the turn of the 80's and 90's of the last century, scientists of the Physical Institute after P.N. Lebedev and Earth Physics Institute (Russia) had developed a concept of a new promising area of seismology: using a signal from elastic vibrations in the acoustic frequency band for earthquake forecasting. These elastic vibrations are generated under local ionization influence, which is formed at the moment when passing of cosmic radiation penetrating particles happens. These particles are high-energy muons and neutrinos, which pass through seismically tense environment in the deeper layers of Earth. The basic idea of this method is illustrated by Fig.3.

Earth crust sounding by a beam of penetrating energetic muons and neutrino enables us to conduct direct monitoring of the lithosphere internal state at the depth of 1-10 km that is the closest to the zone of earthquake sources formation. Together with acoustic monitoring of deep environment response on muon beam trigger action, such sounding represents a unique method of direct penetration in the earthquake zone vicinity. Every individual measuring at the muon monitoring is local and all measurements together allow us control considerable volume of the 
earthquake zone. The size of the zone depends from the sound receiving devices sensitivity, acoustic noise level and installation square for muon flow detection sensitivity.

Acoustic detector signals are recorded in a special room (Fig 4). The room is located at the upper edge of the well. The signal from the microphone through the amplifiers is transmitted to the computer. The whole device is supplied with energy from solar batteries.

Nowadays, the Tien Shan shower installation modification is completed and it started EAS regular registration.

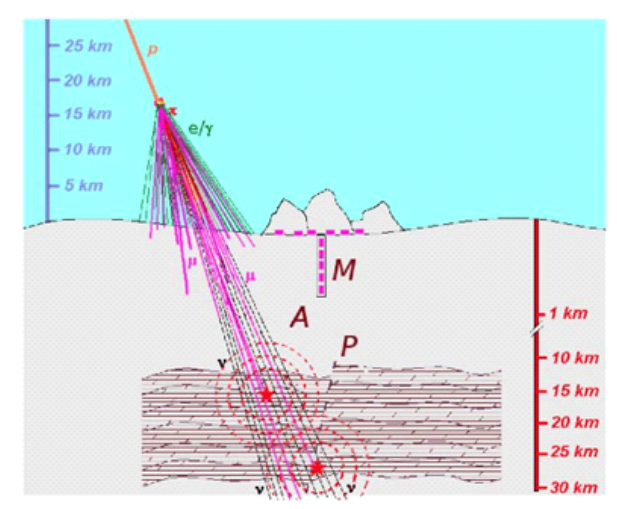

Fig. 3. Model of deeper seismic sounding of Earth's crust on the basis of muons from the energetic EAS trunk: $\mathrm{P}$ is a deep fault zone; $\mathrm{A}$ is elastic vibrations are generated in the seismic stretch environment under local ionization influence from muons passing and propagated as acoustic wave; $M$ is a sensible microphones system.

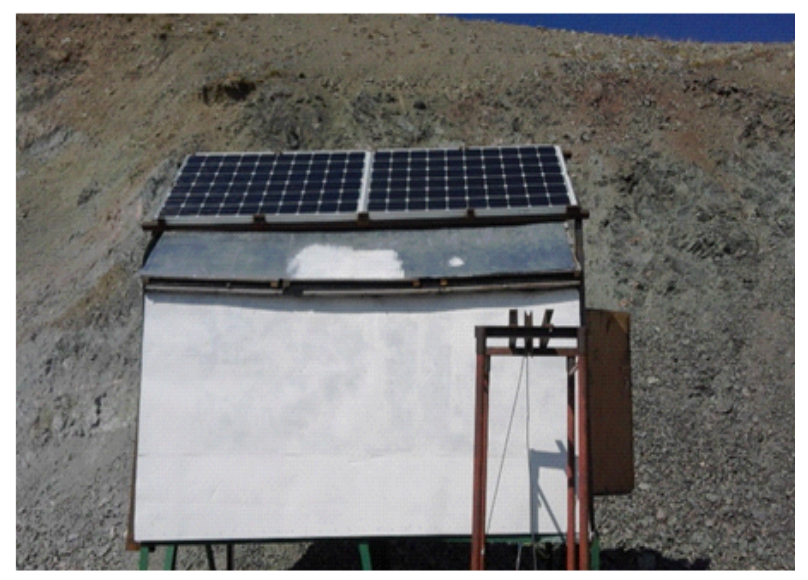

Fig.4. Special room with solar panels installed in its roof

\section{Conclusion}

Today, in high-energy physics there are several strongly different phenomenological models of the hadronic interactions are used, each of which claims for adequate description of hadron-nucleus interaction at the extra-high energy. Their difference leads to significantly 
diverse conclusions about nature of coplanar generation of the most energetic particles that are parts of the extensive air shower (EAS) trunks. The latter cannot be described by the hadronic interactions conventional models, i.e. events of "centaur" or "anti-centaur" types with the anomalous high part energy, released in the charged component, or on the contrary, in the neutral component. Observation of abnormally weak absorbing hadrons permitted to raise a question about the cosmic rays (CR) long-range nuclear-interacting component in the region of the spectra breaking in the mass composition of the primary cosmic rays (PCR). The latter consequently resulted from solving an inverse problem, which is recovering spectra parameters and PCR composition by EAS observed characteristics.

\section{References}

[1] P. Tribedy 2017 Journal of Physics: Conference Series Ser. 832, 012013.

[2] Kroemer O. 2009 Proceedings of the 31st ICRC.- p. 25.

[3] Adamson P. et al. 2007 Phys. Rev. -Vol. D76. -P. 052003.

[4] R.U. Beisembayev, O.D. Dalkarov, T.Kh. Sadykov et.al. 2016 Nuclear Instruments and Methods in Physics Research A. V.832 - p.158-178.

[5] G. V. Kulikov and G. B. Khristiansen. 1959 Soviet Physics JETP 35(8):441-444.

[6] J. Abraham, Pierre Auger Collaboration 2010 Physics Letters B - V685. - p. 239-246.

[7] B A Iskakov et al 2019 J. Phys.: Conf. Ser. 1337012004 